\title{
RstB2 protein, the DNA binding protein of CTX $\Phi$ phage from $V$. cholerae: current status and pending research
}

\begin{abstract}
The phage-encoded protein RstB is required for $\mathrm{CTX} \phi$ phage integration, a lysogenic filamentous phage that contains the genes encoding cholera toxin, the main virulence factor of Vibrio cholerae. This mechanism of integration is catalyzed by the host-encoded tyrosine recombinases, XerC and XerD and some phage-encoded and host-encoded factors. This paper summarizes the results reported so far, of some bioinformatic and biochemical studies of the protein RSTB. Moreover, it has also been informed about ongoing bioinformatic studies which may contribute to clearing up the role of this protein in phage integration CTX $\phi$.
\end{abstract}

Keywords: RstB2 protein, vibrio cholerae, CTXФ phage, DNA-binding protein, singlestranded DNA, double-stranded DNA
Volume I Issue 2 - 2014

\author{
Alina Falero, Rafael Fando \\ Department of Biochemistry, National Center for Scientific \\ Research, Cuba
}

Correspondence: Alina Falero Morejon, Department of Biochemistry, National Center for Scientific Research, Ave 25 and 158, Cubanacan, Playa, PO Box 6214, Havana, Cuba, Tel 5372085236,Email alina.falero@cnic.edu.cu

Received: June II, 20I4 | Published: June 26, 2014
Abbreviations: SSB, single-stranded DNA binding; ssDNA, single-stranded DNA; dsDNA, double-stranded DNA

\section{Introduction}

Cholera is a preventable and easily treatable disease that has been eliminated in industrialized countries but is still present in Africa, Southeast Asia, and currently, it was spread in the Latin America and Caribbean region. It requires immediate treatment because it can cause death within hours, even in previously healthy people. Cholera is caused by infection of the intestine with the Gram-negative bacterium Vibrio cholerae which acquires the virulence factor via lysogenic conversion by a filamentous phage, CTX $\Phi$ carrying the cholera toxin genes. ${ }^{1}$ This unusual lysogenic filamentous phage can either replicate as a plasmid in the absence of the СТХФ chromosome integration site or site-specifically integrate into Vibrio cholerae chromosomes. ${ }^{1,2}$ Lysogenic conversion results from the exchange of a single pair of strands between attachment site on the genome and the bacterial chromosome of one or the other of the two circular chromosomes of $V$. cholerae, which is catalysed by the XerCD recombinases. ${ }^{3-5}$ The mechanism of СТХФ integration into the $V$. Cholerae chromosome has been widely investigated. ${ }^{3-8}$ It has been proven that integration is catalyzed by recombinases encoded by XerCD genes, ${ }^{6}$ using as a substrate the genomic ssDNA of the phage for recombination with the bacterial chromosomal dif1 site. ${ }^{6}$ The genomic ssDNA must fold into stem-loop structure, creating a small region of duplex DNA that is target of the site-specific recombinases. ${ }^{2}$ Like the tyrosine recombinases, the phage-encoded protein RstB and some phage-encoded and hostencoded factors are required for СТХФintegration. ${ }^{3,9}$ Among hostencoded factors, it was found that some of the host proteins determine the catalytic state of the Xer recombinases. ${ }^{10,11}$ This recombination generates either a single CTX $\Phi$ prophage or tandem prophages, which can serve as a template for production of extra chromosomal phage DNA. Unlike other phage site-specific integration mechanisms, CTX $\phi$ integration is irreversible. ${ }^{3}$

As above mentioned, the phage-encoded protein RstB is required for CTX $\phi$ integration; hence it would make sense deepen about its role in the integration process, just to try to control this deadly disease, if possible. However, until we know, there are no abundant papers related with the biochemical study of this protein nowadays.

The first report about biochemical characterization demonstrated that RstBis a DNA binding protein; ${ }^{12}$ because of its gene position and size homology to other genes encoding single-stranded DNA binding (SSB) proteins in other filamentous phages. ${ }^{13}$ Although it preferentially binds ssDNA, it is surprising its capacity to interacting with dsDNA, following similar kinetic reaction in the protein-DNA complex formation. ${ }^{14}$ In fact, RstB is the first protein of a filamentous phage to show affinity for both ds and ssDNA in vitro. Also purification methods for both, recombinant RstB-His and the tag-less RstB native proteins were described, using E. coli as the host. These methods enable obtaining sufficient amounts of protein to carry out further biochemical studies focused at clearing up the role of the protein in the integration process of the phage..$^{2}$ Until we know, there are no other reported other expression vectors for this protein. Recently, we reported that RstB occurs mostly as a monomeric species in solution and it is stable over time, because it retained its ssDNA-binding activity after a year of storage at $-20^{\circ} \mathrm{C}$. Moreover, it was found that its C-terminal His-tail promoted oligomerization and decreased its isoelectric point from 8.52 to 6.47 . On the other hand, bioinformatics analyses of the amino acid sequence of RstB predicted its secondary structure, suggesting the presence of an OB-fold, characteristic of SSB proteins. At the same time, the C-terminal of the protein was predicted as disordered, flexible and exposed, like other SSBs, which may be physiologically relevant during СТХФ integration. Interestingly, some conserved amino acids were found at structurally or functionally important relative positions ${ }^{14}$ as compared with $\mathrm{gVp}$ of M13 filamentous phage, a related to СТXФ phage. ${ }^{1}$ Nevertheless, the three-dimensional structures of the RstB and protein-DNA complex have not been reported yet, to our knowledge.

The three-dimensional structure of the proteins is the key to know how the protein carries out its biological function. ${ }^{15}$ Its experimental determination is based on expensive and time-consuming methods, such as X-ray crystallography, Nuclear Magnetic Resonance 
spectroscopy and Circular Dichroism spectroscopy. However, several computational methods using sequence and/or structural information can be used to propose the three-dimensional structure of both, the RstB protein and the protein-DNA complex. Knowledge of both structures allows us to predict the amino acid residues involved in DNA binding site and binding specificity which is critical for understanding the mechanism of gene regulation and would help to understand the recognition mechanism of protein-DNA complexes. ${ }^{16}$ In addition, conformational changes, functional classes and biophysical features of bound proteins and stability of the complex can be predicted. Such information may be used to address experimental studies such as sitedirected mutagenesis for functional characterization of this protein.

Due to its binding to both, ssDNA and dsDNA with similar kinetic reaction, we have previously suggested that RstB may be a noncanonical filamentous phage SSB protein of an unusual filamentous phage, such as СТХ $\Phi .{ }^{14}$ Therefore, it would be of interest testing the affinity of this protein by the DNA triplex, which could give more light to outstanding questions related to СТХФ integration.

On the other hand, it was found that the shape of DNA is the determining factor in the cellular function. ${ }^{17}$ Recently it was reported that a small fraction of genomic DNA can adopt the non-canonical B-DNA structure or unusual DNA structure. The unusual DNA structures like DNA-hairpin, cruciform, Z-DNA, triplex and tetraplex are represented as hotspots of chromosomal breaks, homologous recombination and gross chromosomal rearrangements since they are prone to the structural alterations..$^{18}$ These repetitive DNA motifs are abundant in the genomes of various species, which are not random and often co-localize with sites of chromosomal breakage associated with genetic diseases. ${ }^{19}$

Therefore, based on the knowledge described above we think that it would be very useful prove if CTXФ phage have the capacity to adopt non-canonical (i.e. non-B) DNA structures like triplex DNA, which could be addressed by several experimental and bioinformatic methods reported before. ${ }^{20-24}$

Thus, considering the above explained, we are carrying out some bioinformatics analyses to predict the three-dimensional structures of both, from the RstB protein and the protein-DNA complex that allows us getting all information possible to pave the way for further studies addressed at clearing up the role of RstB protein in the integration process.

\section{Acknowledgements}

None.

\section{Conflict of interest}

The author declares no conflict of interest.

\section{References}

1. Waldor MK, Mekalanos JJ. Lysogenic conversion by a filamentous phage encoding cholera toxin. Science. 1996;272(5270):1910-1914.

2. Moyer KE, Kimsey HH, Waldor MK. Evidence for a rolling-circle mechanism of phage DNA synthesis from both replicative and integrated forms of CTXphi. Mol Microbiol. 2001;41(2):311-323.

3. Huber KE, Waldor MK. Filamentous phage integration requires the host recombinases XerC and XerD. Nature. 2002;417(6889):656-658.

4. Campbell AM. Chromosomal insertion sites for phages and plasmids. $J$ Bacteriol. 1992;174(23):7495-7499.
5. Waldor MK, Rubin EJ, Pearson GD, et al. Regulation, replication, and integration functions of the Vibrio cholerae CTXphi are encoded by region RS2. Mol Microbiol. 1997;24(5):917-926.

6. Val ME, Bouvier M, Campos J, et al. The single-stranded genome of phage CTX is the form used for integration into the genome of Vibrio cholerae. Mol Cell. 2005;19(4):559-566.

7. Davis BM, Waldor MK. Filamentous phage linked to virulence of Vibrio cholerae. Curr Opin Microbiol. 2003;6(1):35-42.

8. Nash HA. Site-specific recombination: integration, excision, resolution, and inversion of defined DNA segments. In: Neidhardt FC, editor Escherichia coli and Salmonella. USA: ASM Press; 1996. p. 2363-2376.

9. McLeod SM, Waldor MK. Characterization of XerC- and XerDdependent CTX phage integration in Vibrio cholerae. Mol Microbiol. 2004;54(4):935-947.

10. Colloms SD, Bath J, Sherratt DJ. Topological selectivity in Xer sitespecific recombination. Cell. 1997;88(6):855-864.

11. Bregu M, Sherratt DJ, Colloms SD. Accessory factors determine the order of strand exchange in Xer recombination at psi. EMBO J. 2002;21(14):3888-3897.

12. Falero A, Caballero A, Ferran B, et al. DNA binding proteins of the filamentous phages CTXF and VGJF of Vibrio cholerae. J Bacteriol. 2009;191(8):5873-5876.

13. Campos J, Martinez E, Suzarte E, et al. VGJ phi, a novel filamentous phage of Vibrio cholerae, integrates into the same chromosomal site as CTX phi. J Bacteriol. 2003;185(19):5685-5696.

14. Falero A, Marrero K, Trigueros S, et al. Characterization of the RstB2 protein, the DNA-binding protein of CTXF phage from Vibrio cholerae. Virus Genes. 2014;48(3):518-527.

15. Habibi M, Eslahchi C, Sadeghi M, et al. The interpretation of protein structures based on graph theory and contact map. Open Access Bioinformatics. 2010;2:127-137.

16. Nagarajan R, Ahmad S, Gromiha M. Novel approach for selecting the best predictor for identifying the binding sites in DNA binding proteins. Nucleic Acids Res. 2013;41(16):7606-7614.

17. Rajeswari MR. DNA triplex structures in neurodegenerative disorder, Friedreich's ataxia. J Biosci. 2012;37(3):519-532.

18. Vasquez KM, Glazer PM. Triplex-forming oligonucleotides: principles and applications. $Q$ Rev Biophys. 2002;35(1):89-107.

19. Zhao J, Bacolla A, Wang G, et al. Non-B DNA structure-induced genetic instability and evolution. Cell Mol Life Sci. 2010;67(1):43-62.

20. Rajeswari MR. Tryptophan intercalation in G, C containing polynucleotides: $\mathrm{Z}$ to $\mathrm{B}$ conversion of poly $[\mathrm{d}(\mathrm{G}-5 \mathrm{M} \mathrm{C})]$ in low salt induced by a tetra peptide. J Biomol Struct Dyn. 1996;14(1):25-30.

21. Mills M, Arimondo PB, Lacroix L, et al. Energetics of stranddisplacement reaction in triple helices: a spectroscopic study. J Mol Biol. 1999;291(5):1035-1105.

22. Jain A, Rajeswari MR, Ahmed F. Formation and thermodynamic stability $(\mathrm{R} * \mathrm{R} * \mathrm{Y})$ DNA triplex in GAA/TTC repeats associated with Freidreich's Ataxia. J Biomol Stuct Dyn. 2002;19(4):691-699.

23. Lexa M, Martinek T, Burgetova I, et al. A dynamic programming algorithm for identification of triplex-forming sequences. Bioinformatics 2011;27(18):2510-2517.

24. Hon J, Martinek T, Rajdl K, et al. Triplex: an R/Bioconductor package for identification and visualization of potential intramolecular triplex patterns in DNA sequences. Bioinformatics. 2013;29(15):1900-1901. 